\title{
Power Distribution Network Based on Digital 3D Twin Panoramic Modeling
}

\author{
Zhan Chao ${ }^{1}$, Luo Yizhao ${ }^{1}$, Li Ronggui ${ }^{1}$, Gao Jun ${ }^{2}$, Zhang $\mathrm{Mi}^{2}$ \\ ${ }^{1}$ Fujian Netpower Technology Development Co.,Ltd.,Fujian Fuzhou,350003 \\ ${ }^{2}$ Shenzhen Turingsmart Technology Co.Ltd., Guangdong Shenzhen, 518000
}

\begin{abstract}
Digital twins refer to virtual digital expressions constructed in virtual space that can characterize characteristics, the formation process and behavior of natural entities, and it has the characteristics of multi-physics, multi-scale and probability. This article mainly introduces the application research of digital 3D twin panorama modeling in power distribution network. This paper proposes the average current method to calculate the line loss of the power distribution network, so that the accuracy is guaranteed. In addition, the digital three-dimensional panoramic modeling method is used to optimize the grid system, and the data structure is used to calculate the power distribution network. Analyze the topological structure of the power grid, and then obtain the calculation method of the distribution network line loss. The experimental results in this paper show that the rendering efficiency of power grid results has increased by $17 \%$ based on the digital $3 \mathrm{D}$ twin panorama modeling, and the method and algorithm for matching video and panorama image are proposed, which solves the problem of video rotation angle on the panorama connection, so that The smoothness of panoramic roaming is improved by $23 \%$.
\end{abstract}

Keywords: Digital twin, panoramic modeling, power distribution network, 3D modeling

\section{Introduction}

Electric power distribution network refers to the electric energy meter that the power loss generated by the station during the operation of the power grid or the substation reaches the user during the power transmission process [1]. The power loss rate is a measure of the degree of loss in the energy transfer process. It fully reflects the design, design, operation and management level of the power system, and is an important economic and technical indicator of the operation of the power grid [2]. The theoretical line loss calculation is to use a specific mathematical calculation model to calculate the power loss in the grid loss. According to the network structure, network components, operating parameters and load conditions, and thereafter, calculate the theoretical loss rate according to the ratio of power loss to power supply [3- 4]. The theoretical line loss rate is an important basis for power supply companies to formulate reasonable line loss management indicators. At the same time, the calculation of theoretical line loss is also an important basis for determining 
whether the network design is scientific and reasonable during the design process. Therefore, the theoretical calculation of distribution network line loss is of great significance to power supply companies [5].

Chakshu NK proposed a method that can detect the severity of carotid artery stenosis through face video with the help of coupled blood flow and blood flow. This semi-active digital twin model is an attempt to correlate the non-invasive video of the patient's face with the percentage of carotid artery occlusion [6]. The pulsating nature of the blood flow through the carotid artery causes subtle vibrations in the head. This vibration is a potential indicator of the severity of carotid artery stenosis, and he has proposed a head vibration model that is related to the force generated by blood flow with or without obstruction. However, due to many unstable factors in the head, the experimental results are not very accurate [7].

The innovation of this paper is that this paper proposes a boundary fusion method to solve the problem of obvious gaps caused by the use of LOD technology to divide the panorama into hierarchical blocks, which can make the color transition [8]. And proposed the use of ray intersection judgment and block loading processing method to accelerate the loading of panoramic images, so that the three-dimensional panoramic digital interactive exhibition platform is more smooth [9-10].

\section{Digital Three-Dimensional Panoramic Modeling Power Distribution Network Calculation Method}

\subsection{Calculation Method of Power Distribution Network Line Loss}

Research and analysis of various calculation models show that the average current method can obtain better calculation accuracy, and the required calculation parameters are usually easy to obtain, which is more suitable for the theoretical line loss calculation needs of rural power grids. At the same time, because the calculation model is convenient for multiple power sources The theoretical line loss of the distribution network is calculated, and it is listed as the first choice for calculating the theoretical line loss of the rural distribution network with multiple small hydropower stations, which is more in line with the actual situation of the southern rural power grid with more small hydropower. The disadvantage is that it is not easy to obtain the load characteristic coefficient value of the load point. For this, the average current method can be used to calculate the load characteristic coefficient of the transformer from the typical load ratio of various load transformers through equation (1), so that it can further weaken its influence calculation The prerequisite of accuracy ensures the accuracy of calculation. Therefore, it was decided to use the average current method calculation model as the theoretical line loss calculation model used in this system.

$$
T=\sqrt{0.2 / f+0.8}
$$

The average current method is also called the shape factor method, which uses the equivalent ratio between the root mean square current method and the average current to calculate the power loss, as well as the root mean square current. The basic idea of the average current method is: the power loss caused by the average current flowing on the line is equal to the actual power loss and the load is simultaneously loaded. The calculation types are as follows:

$$
\Delta B=3 I_{d l}^{2} T^{2} R t \times 10^{-3}
$$


Among them, $\Delta B$ is the power loss of power grid components, $I_{d l}^{2}$ is the average current flowing through the components, $K$ is the load shape factor of the components, and $t$ is the running time.

\subsection{Digital 3D Power Grid Panoramic Modeling Method}

\section{(1) Hierarchical grid construction}

Based on rich geographic information data, the required power equipment models such as substations, towers, insulator strings, foundations, spacers, shock-proof hammers, etc. are unified into the library; secondly, the hierarchical grid details are organized and stored in the library in units of loops according to business logic relationships. Preliminary detection prompts and verification modifications are made to unreasonable data, and then the power engine is used to drive the construction of three-dimensional grid results; after the construction of the grid results is completed, the results need to be further optimized, including dynamic wire index creation and LOD optimization.

(2) LOD rendering technology

LOD (Level of Detail Model Technology) rendering is to build a face model from the original polyhedron, and based on the distance and size elimination algorithm, the constant number of triangles algorithm, the mesh simplification algorithm based on edge folding, the material merging algorithm, and the geometry data block Means such as sharing between texture blocks and sharing between texture blocks reduce the number of topologies and structural edges in the coating structure to reduce data complexity and IO efficiency and affect visual effects, thereby improving multi-party data access and performance.

(3) Catenary algorithm

In the transmission line, due to the rigidity of the wire, the wires between adjacent towers will sag to a certain extent, forming an arc; at the same time, the choice of the sag formula is also related to the error of the wire use stress and the cross of the wire pairs. The problem of span error. The parametric models of wires, cable shafts, tunnels and cable lines are created by dynamic indexing of quadtrees, and the data is tiled according to the scale range, and then displayed in the three-dimensional scene through real-time windows to achieve efficient rendering and Load, thus ensuring the stability of the 3D scene.

\section{Digital Twin Model Establishment}

Through the operation and analysis of the virtual digital twin space, it is possible to monitor, diagnose, predict and control the process, state and behavior of natural entities without the creation of natural entities in the real environment. With the deepening of people's understanding of the world and the enhancement of their ability to simulate the natural world, digital twins have increasingly enhanced their ability to describe nature, but they cannot completely replace natural entities in certain key connections.

According to the theory of product life cycle management, natural entities change dynamically with the stage of the product. Since digital twins are mirror images of physical entities in virtual space, digital twins are also changing dynamically.

The mirror relationship model that describes the digital dual relationship between the physical entity and the virtual space in the physical space usually includes the following four steps: based on the data mechanism and scientific knowledge of the physical entity, a digital twin is created in the virtual space; it will use the control unit, Sensors, etc. The collected functional entity state data and historical operation and maintenance data should be dynamically synchronized with the digital twin system; and re-optimized; a virtual environment reflecting the real environment is constructed in the virtual space and the 
optimized digital twin. The simulated natural entity in the virtual environment is in real Behavior in the environment; analysis of simulation results, creating valuable information and providing it to natural entities, improving and optimizing design, construction, operation and maintenance of natural entities. Generally speaking, a virtual space consists of a physical entity and a virtual environment reflecting the actual operating environment, which is also a digital twin of their respective physical spaces.

\section{Power Distribution Network Analysis under the Digital Three-Dimensional Panoramic Model}

\subsection{Analysis of Digital 3D Panoramic Model}

The three-dimensional digitization of power grid engineering realizes the transfer and management of three-dimensional information model data of power grid engineering from the design and infrastructure period to the operation period. It mainly realizes the two major business functions of data management and data application. The process includes design result data transfer, data review, data management, and data Sharing and data display. And can provide data sharing and service interfaces, thereby effectively providing data sharing services and avoiding information islands. The transfer process of the entire power grid project includes a series of operations such as data collection, transfer, review, inspection, management, release, sharing and display; the transferred project data results must be transferred in strict accordance with the transfer specifications, through 3D design, 3D Modeling, laser scanning, oblique photography and other technical means, to carry out three-dimensional modeling of the design schemes, equipment and facilities of each stage of the power grid project, and to conduct data audit and quality inspection of the three-dimensional handover results in accordance with the handover specifications. Digital storage management to ensure the correct construction of a complete digital information model of the power transmission and transformation project infrastructure area.

This article summarizes the evaluations of 100 power grid workers on the digital 3D panorama model. Through analysis, it can be seen that among the 100 workers, 36 workers are very satisfied with the establishment of the digital 3D panorama model, accounting for $36 \%$ of the total. $\%$, and 45 people are generally satisfied with the establishment of the digital 3D panoramic model, accounting for $45 \%$ of the total. Among them, the number of people who are uncertain about the establishment of the digital 3D panoramic model accounted for $9 \%$, and for the digital 3D panoramic model. Only $7 \%$ were dissatisfied with the establishment of the panoramic model. Finally, only 3\% were dissatisfied with music teaching under digital multimedia VR technology. The specific results are shown in Figure 1.

A summary and analysis of the evaluation can be drawn to build a three-dimensional digital platform for power grid engineering, and use big data, cloud computing, Internet of Things, mobile and visualization technology to provide design results data for different stages of power grid engineering design and construction, etc. Carry out the unified and centralized management of three-dimensional digitalization, realize the integrated sharing of design results, build a three-dimensional digital power grid, promote business integration, assist digital design, and effectively manage the three-dimensional design results of power grid projects through digital and three-dimensional visualization, and better service multi-level response, Risk early warning, statistical analysis, etc., to enhance the comprehensive handling capabilities for the safe operation of elevators, and play a positive role in the construction of safe cities and smart cities. 


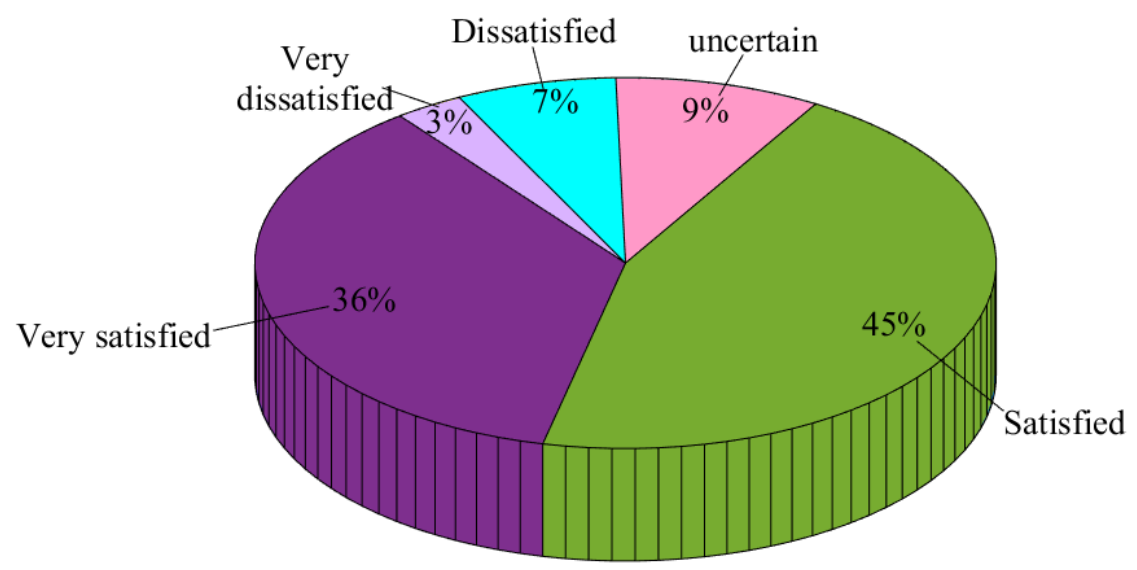

Figure 1. Evaluation of the establishment of a digital 3D panoramic model

\subsection{Algorithm Analysis of Power Distribution Network Line Loss}

This article supplies power to a power supply substation and a small hydropower connected to a distribution network. It means that the active power at the head end of the daily distribution network is $3212 \mathrm{~K}$ var.h, the reactive power is $3132 \mathrm{KW} . \mathrm{h}$, the average line voltage is $10 \mathrm{KW}$, the active power of small power supply is $1642 \mathrm{KW} . \mathrm{h}$, and the reactive power is $1442 \mathrm{Kvar}$.h represents the head of the daily distribution network. Current two, two. The monthly power supply of each distribution transformer is shown in Table 1:

Table 1. Distribution network distribution transformer model and monthly power supply

\begin{tabular}{|c|c|c|}
\hline Name & Model & Active power \\
\hline 1 & SJ-180 & 59325 \\
\hline 2 & SJ-180 & 5434 \\
\hline 3 & SJ-50 & 9322 \\
\hline 4 & SJ-50 & 4523 \\
\hline
\end{tabular}

Comparing the theoretical line loss results calculated in this paper with the Newton power flow method, the following comparison results are obtained, as shown in Figure 2.

Assuming that the result of the Newton power flow method is accurate, it can be seen from the above analysis that the result calculated by the system is closer to the actual value than the traditional equivalent resistance method. The three methods are compared. When the power supply is the same, the Newton power flow method is The bus loss rate is the lowest at $4.89 \%$. The relative error of the average current method and the Newton power flow method is $5.23 \%$, which fully meets the needs of engineering calculations. 


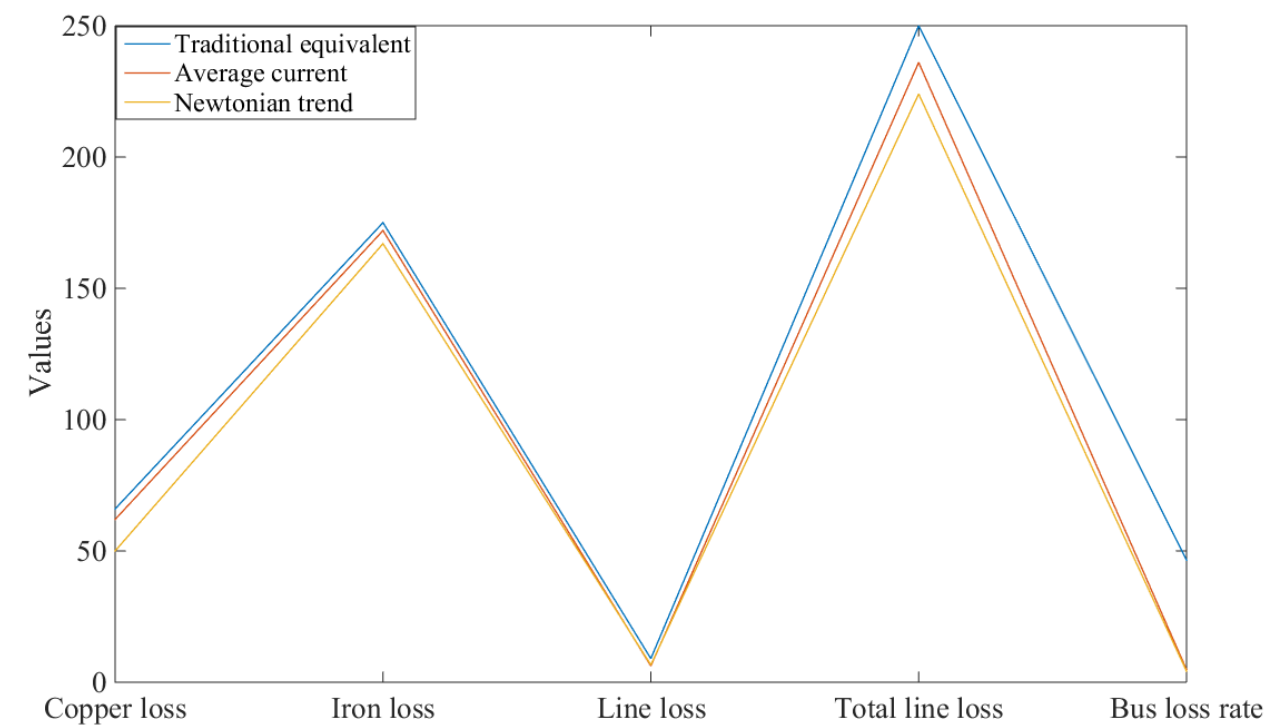

Figure 2. Comparison of results of different line loss calculation methods

\section{Conclusions}

Various applications in the field of power system analysis inevitably require the analysis of the grid topology. Appropriate grid topology description methods and methods have been the focus and difficulty that plagued many applications in this field for a long time, and there is a lack of unified and consistent tools or Technology to describe the power grid is the root cause of the problem, until the emergence of digital 3D twin panoramic modeling, it provides another brand new approach and method for software development in this field.

\section{References}

1. Chakshu N K, Carson J, Sazonov I, et al. A semi-active human digital twin model for detecting severity of carotid stenoses from head vibration-A coupled computational mechanics and computer vision method[J]. Communications in Numerical Methods in Engineering, 2019, 35(5):e3180.1-e3180.17.

2. Lee $\mathbf{J}$. Integration of Digital Twin and Deep Learning in Cyber-Physical Systems: Towards Smart Manufacturing[J]. 2020, 38(8):901-910.

3. Li C , Mahadevan S, Ling Y, et al. Dynamic Bayesian Network for Aircraft Wing Health Monitoring Digital Twin[J]. AIAA Journal, 2017, 55(3):1-12.

4. Zhang H, Liu Q, Chen X, et al. A digital twin-based approach for designing and decoupling of hollow glass production line[J]. IEEE Access, 2017, 5(2017):26901-26911.

5. Hamada M, Takeshita A, Shimizu H, et al. Surgical simulation for dentigerous cyst in the maxillary sinus using full-color 3D salt modeling: Report of a case[J]. Oral \& Maxillofacial Surgery Cases, 2018, 4(1):6-11.

6. Kim H , Han S . Geo-registration of wide-baseline panoramic image sequences using a digital map reference[J]. Multimedia Tools \& Applications, 2017, 76(9):11215-11233.

7. Zeynalian M , Khorasgani M Z . Structural performance of concrete poles used in 
electric power distribution network[J]. Archives of Civil and Mechanical Engineering, 2018, 18(3):863-876.

8. Yan Y, Jiang W, Zou D, et al. Research on mechanism configuration and coordinated control for power distribution network live working robot[J]. Industrial Robot: the international journal of robotics research and application, 2020, 47(3):453-462.

9. Liu Y, Du S, Sheng W . Classification and identification of electric shock current for safety operation in power distribution network[J]. 2020, 5(2):145-152.

10. S. Guo, Z. Zhao, Q. Zhang. Failure rate model for optical fiber cable in electric power communication network based on Weibull distribution function[J]. Power System Protection \& Control, 2017, 45(17):92-99. 